\title{
Myocardial strain analysis with CMR in cardiotoxicity patients using deformation field analysis: Comparison to healthy volunteers and heart transplant patients
}

\author{
Abraham Bogachkov ${ }^{*}$, Kai Lin², Benjamin H Freed ${ }^{3}$, Michael Markl², James C Carr ${ }^{4}$, Jeremy D Collins ${ }^{4}$ \\ From 19th Annual SCMR Scientific Sessions \\ Los Angeles, CA, USA. 27-30 January 2016
}

\section{Background}

Noninvasive monitoring of cardiac function in patients with iatrogenic or environmentally-induced cardiotoxicity is of prime importance as a measure of disease severity. Strain imaging at cardiac MR (CMR) has been previously shown to be a highly robust modality in the detection of early cardiac dysfunction in the heart failure population. However, strain analysis has not previously been applied in the assessment of cardiotoxicity patients. The purpose of this study is to compare radial and circumferential LV strain values in patients with known cardiotoxicity to healthy volunteers and post-heart transplant patients, and to then correlate the cardiotoxicity strain values to calculated LV ejection fractions.

\section{Methods}

Retrospective analysis of CMR images from 11 volunteers (9/11 males, avg. age 51.6), 5 heart transplant patients (5/5 males, avg. age 60.2), and 10 cardiotoxicity patients (3/10 males, avg. age 48.9) were obtained at 1.5 T (MAGNETOM Avanto, Siemens Medical Systems, Erlangen, AG) using GRAPPA factor 2 acceleration. Myocardial strain analysis at CMR was performed using semi-automatic prototype software calculating Lagrangian strain from deformation field analysis (Siemens Corp, Corporate Technology, Princeton, NJ). Left ventricular (LV) midwall average and peak systolic radial and circumferential strains data was calculated. Strain data between groups was compared using univariate analysis of variance (ANOVA) to assess statistical equivalence.

${ }^{1}$ Northwestern University, Feinberg School of Medicine, Chicago, IL, USA Full list of author information is available at the end of the article
Cardiotoxicity patients' strain data was compared to calculated LVEF values using linear regression with associated $R^{2}$ values.

\section{Results}

Mean global peak radial and circumferential strain values and mean average-segmental peak radial and circumferential strain values were calculated for subjects in the 3 cohorts. Mean strain values were found to be lower in cardiotoxicity patients with $\mathrm{EF}<50 \%$ vs. healthy volunteers in both peak radial and circumferential strain in a statistically significant $(\mathrm{p}<0.05)$ manner (Figure 1). All other groups analyzed were found to have statistically equivalent mean strain values, including both subsets of cardiotoxicity patients. The $\mathrm{R}^{2}$ values for linear regression of global peak radial and circumferential strain values vs. EF were 0.61 and 0.76 , and 0.84 and 0.95 for mean average-segmental peak radial and circumferential strain, respectively (Figure 2).

\section{Conclusions}

Radial and circumferential LV strain values in patients with known cardiotoxicity were found to be lower than volunteers in a statistically significant manner when using a semi-automatic prototype software calculating Lagrangian strain from deformation field analysis. Furthermore, strain values were found to correlate strongly with LVEF, a well-established clinical marker of cardiotoxicity, particularly in the mean average-segmental peak radial and circumferential strain values. Continued work is necessary to elucidate a more defined clinical role for CMR-calculated strain in the cardiotoxicity patient population. 
A HDA Global Max Radial Strain

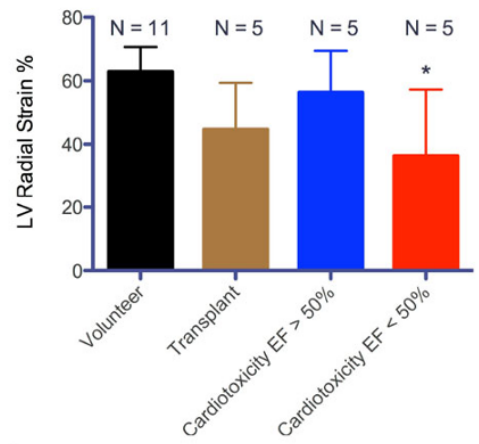

C HDA Global Max Circumferential Strain

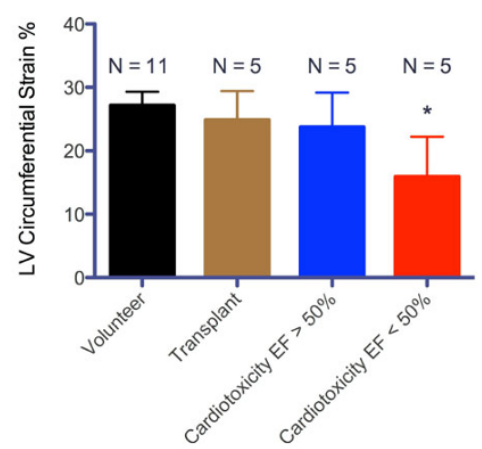

B HDA Global Average Max Radial Strain

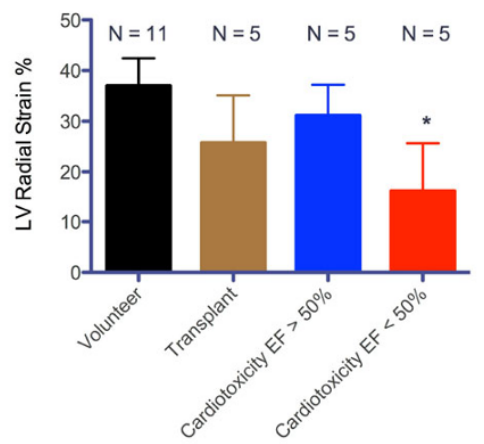

D HDA Global Average Max Circumferential Strain

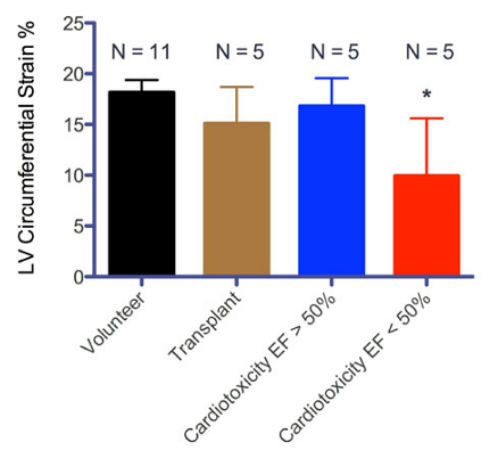

Figure 1 Average left ventricular strain as calculated by a semi-automatic prototype software calculating Lagrangian strain from deformation field analysis with $\mathbf{9 5 \%} \mathrm{Cl}$. A - mean peak radial strain value amongst the 3 groups; $\mathbf{B}$ - mean of average segmental peak radial strain value amongst the 3 groups; $\mathbf{C}$ - mean peak circumferential strain value amongst the 3 groups; $\mathbf{D}$ - mean of average segmental peak circumferential strain value amongst the 3 groups. ${ }^{*}$ indicated statistically significant $(p<0.05)$ difference from volunteer group.

A HDA global max radial strain vs. EF

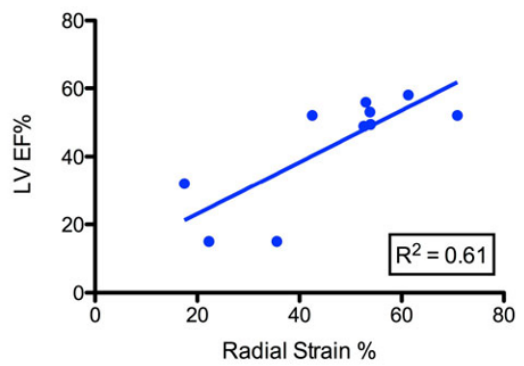

C HDA global max circumferential strain vs. EF

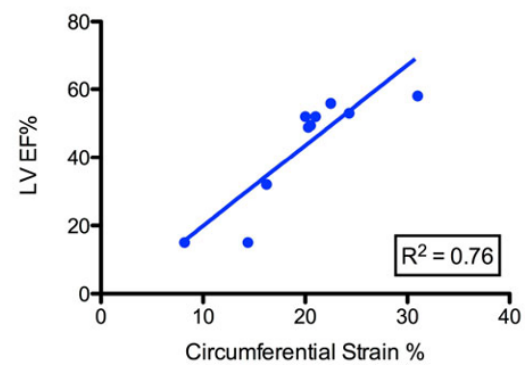

B

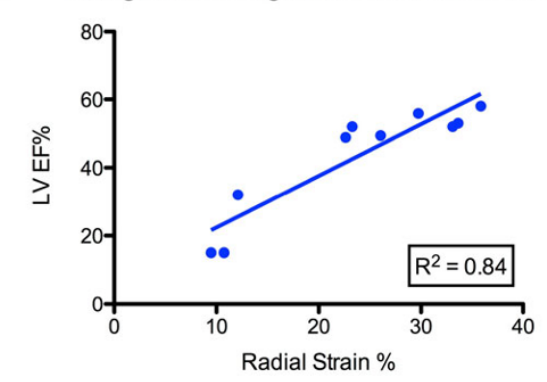

D HDA global Average max circumferential strain vs. EF

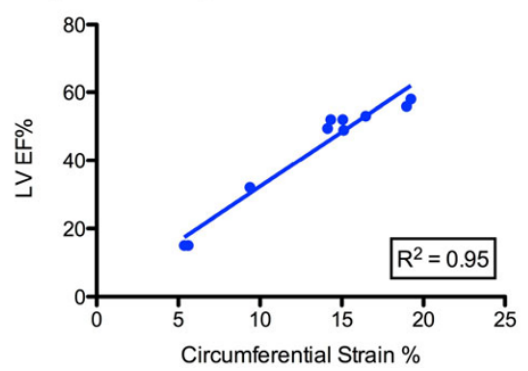

Figure 2 A - linear regression of mean peak radial strain value from the cardiotoxicity group vs. calculated LV EF; B - linear regression of mean of average segmental peak radial strain values from the cardiotoxicity group vs. calculated LV EF; C - linear regression of mean peak circumferential strain value from the cardiotoxicity group vs. calculated LV EF; D - linear regression of mean of average segmental peak circumferential strain values from the cardiotoxicity group vs. calculated LV EF. 


\section{Authors' details}

${ }^{1}$ Northwestern University, Feinberg School of Medicine, Chicago, IL, USA.

${ }^{2}$ Cardiovascular MR R\&D, Siemens Healthcare, Chicago, IL, USA. ${ }^{3}$ Cardiology, Northwestern University, Chicago, IL, USA. ${ }^{4}$ Radiology, Northwestern

University, Chicago, IL, USA.

Published: 27 January 2016

doi:10.1186/1532-429X-18-S1-W30

Cite this article as: Bogachkov et al:: Myocardial strain analysis with CMR

in cardiotoxicity patients using deformation field analysis: Comparison

to healthy volunteers and heart transplant patients. Journal of

Cardiovascular Magnetic Resonance 2016 18(Suppl 1):W30.

Submit your next manuscript to BioMed Central and take full advantage of:

- Convenient online submission

- Thorough peer review

- No space constraints or color figure charges

- Immediate publication on acceptance

- Inclusion in PubMed, CAS, Scopus and Google Scholar

- Research which is freely available for redistribution

Submit your manuscript at www.biomedcentral.com/submit 